\title{
Impact of home care versus alternative locations of care on elder health outcomes: an overview of systematic reviews
}

Laura Boland ${ }^{1}$, France Légarée ${ }^{*}$, Maria Margarita Becerra Perez ${ }^{2}$, Matthew Menear ${ }^{2}$, Mirjam Marjolein Garvelink², Daniel I. Mclsaac ${ }^{3}$, Geneviève Painchaud Guérard ${ }^{2}$, Julie Emond ${ }^{4}$, Nathalie Brière ${ }^{5}$ and Dawn Stacey ${ }^{6,7}$

\begin{abstract}
Background: Many elders struggle with the decision to remain at home or to move to an alternative location of care. A person's location of care can influence health and wellbeing. Healthcare organizations and policy makers are increasingly challenged to better support elders' dwelling and health care needs. A summary of the evidence that examines home care compared to other care locations can inform decision making. We surveyed and summarized the evidence evaluating the impact of home care versus alternative locations of care on elder health outcomes.

Methods: We conducted an overview of systematic reviews. Data sources included MEDLINE, the Cochrane Library, EMBASE, and CINAHL. Eligible reviews included adults 65+ years, elder home care, alternative care locations, and elder health outcomes. Two independent reviewers screened citations. We extracted data and appraised review quality using the Assessing the Methodological Quality of Systematic Reviews (AMSTAR) checklist. Results were synthesized narratively.

Results: The search yielded 2575 citations, of which 19 systematic reviews were eligible. Three hundred and forty studies with 271,660 participants were synthesized across the systematic reviews. The categories of comparisons included: home with support versus independent living at home ( $n=11$ reviews), home care versus institutional care $(n=3$ reviews), and rehabilitation at home versus conventional rehabilitation services $(n=7$ reviews). Two reviews had data relevant to two categories. Most reviews favoured home with support to independent living at home. Findings comparing home care to institutional care were mixed. Most reviews found no differences in health outcomes between rehabilitation at home versus conventional rehabilitation services. Systematic review quality was moderate, with a median AMSTAR score of 6 (range 4 - 10 out of 11).

Conclusions: The evidence on the impact of home care compared to alternative care locations on elder health outcomes is heterogeneous. Our findings support positive health impacts of home support interventions for community dwelling elders compared to independent living at home. There is insufficient evidence to determine the impact of alternative care locations on elders' health. Additional research targeting housing and care options for the elderly is needed.
\end{abstract}

Keywords: Location of elderly care, Health, Overview of reviews, Shared decision making, Decision Support

\footnotetext{
* Correspondence: France.Legare@mfa.ulaval.ca

${ }^{2} \mathrm{CHU}$ de Québec Research Centre-Université Laval site Hôpital St-Francois

d'Assise, 10 Rue Espinay, Quebec G1L 3 L5, Canada

Full list of author information is available at the end of the article
}

(c) The Author(s). 2017 Open Access This article is distributed under the terms of the Creative Commons Attribution 4.0 International License (http://creativecommons.org/licenses/by/4.0/), which permits unrestricted use, distribution, and reproduction in any medium, provided you give appropriate credit to the original author(s) and the source, provide a link to the Creative Commons license, and indicate if changes were made. The Creative Commons Public Domain Dedication waiver (http://creativecommons.org/publicdomain/zero/1.0/) applies to the data made available in this article, unless otherwise stated. 


\section{Background}

Location of care (LOC) for the elderly has become an increasingly important societal issue [1]. With a demographic shift towards an aging population with complex healthcare needs, healthcare systems face challenges in providing long-term care for elders [2, 3]. One such challenge is matching the elders' health and wellbeing needs to the environment. The ecological theory of aging suggests that the environment influences elders' functional status. Promoting optimal outcomes requires a goodness of fit between the elders and the environment [4]. For example, the person-environment fit can positively or negatively influence health outcomes if personal competencies are well-suited, or alternatively, poorly matched to environmental demands. The World Health Organization's report on Aging and Health highlighted the importance of environmental influence on functioning and research has shown that elders' opportunity to build and maintain functional ability is enhanced when they live in an environment that addresses their needs [5]. As such, decision makers (e.g., policy makers, patients, family members, and healthcare providers) are challenged to better consider the impact of the environment, or LOC, on elders' health and wellbeing outcomes.

To make a well-informed decision, stakeholders need to know their options (i.e., available LOCs), the benefits and harms of each option, and how these benefits and harms relate to their personal situation [6]. Common long-term LOC options include home care and institutional care [7]. Home care typically includes independent living at home or living at home with supports and/or modifications to enhance health and independence. Institutional LOCs typically refer to nursing home care or skilled nursing care facilities. Elders and caregivers have identified deciding about moving from home to an alternative LOC as one of the most difficult decisions they face $[8,9]$. This decision is complicated by the evolving contextual factors relating to the care situation such as elder health status, characteristics of the caregivers, and physical environment [8]. Access to evidence describing the impact of home care versus an alternative LOC on elder health and wellbeing could help inform decision making.

Several systematic reviews have examined the impact of LOC on elder health outcomes. However, these reviews tend to narrowly focus on the health impact of a specific intervention (e.g., fall reduction interventions, palliative care, case management) delivered at different care locations. Although individual reviews provide important contributions, a single document that summarizes a broader range of findings will improve access to the literature and help inform stakeholders' decisions about elder LOC. Additionally, it will highlight important knowledge gaps and help prioritise future research questions. Therefore, we summarized available research findings that examined the impact of home care versus alternative LOCs on elder health and well-being.

\section{Methods \\ Design}

We conducted an overview of systematic reviews. An overview design attempts to survey, summarize, and describe the literature allowing findings to be efficiently compared and contrasted [10]. The paper is reported according to the Preferred Reporting Items for Systematic Reviews and Meta-Analyses (PRISMA) guidelines [11]. However, we did not register our protocol in PROSPERO [12]. We initially piloted our protocol using a single database [13]. Changes from the original protocol to the current study included limiting the population from elders and caregivers, to elders only. Our protocol can be available to readers upon request to the corresponding author.

\section{Review eligibility criteria}

The PICOS framework guided systematic review eligibility criteria (Table 1). We included systematic reviews that examined: adults aged 65 or greater, home care, alternative LOCs, and health outcomes (e.g., physical and mental health, morbidity, mortality, functional status and dependence, activities of daily living, quality of life, falls, etc.). We defined systematic reviews as a review of the evidence that included a clear research question, used systematic methods to identify, select and appraise the primary research, and extracted and

Table 1 Inclusion and Exclusion Criteria

\begin{tabular}{|c|c|c|}
\hline Criteria & Included & Excluded \\
\hline Population & Adults 65 years old or greater & \\
\hline Interventions & Home care & $\begin{array}{l}\text { Acute hospital stay } \\
\text { Respite or short term } \\
\text { stay (<3 months) }\end{array}$ \\
\hline Comparison & Alternative long-term LOCs & $\begin{array}{l}\text { Acute hospital stay } \\
\text { Respite or short term } \\
\text { stay (<3 months) }\end{array}$ \\
\hline Outcomes & $\begin{array}{l}\text { Elders' health and } \\
\text { wellbeing outcomes, including } \\
\text { but not limited to: physical and } \\
\text { physiological health, morbidity, } \\
\text { mortality, functional status and } \\
\text { dependence, activities, quality } \\
\text { of life, and falls. }\end{array}$ & \\
\hline Design & $\begin{array}{l}\text { Systematic reviews, with or } \\
\text { without meta-analyses, of } \\
\text { studies that reviewed } \\
\text { randomised controlled trials, } \\
\text { controlled clinical trials, controlled } \\
\text { before and after studies, descriptive } \\
\text { studies, cohort studies, } \\
\text { retrospective studies, and cross- } \\
\text { sectional studies. }\end{array}$ & $\begin{array}{l}\text { Non-systematic review } \\
\text { studies } \\
\text { Conference abstracts } \\
\text { Individual studies }\end{array}$ \\
\hline
\end{tabular}


analysed/synthesized data from included studies [14]. We did not restrict systematic reviews types (e.g. metaanalysis, narrative), dates, or language of publication. Non-systematic reviews, individual studies, and abstracts were excluded. Participant's age had to be reported in the results section of the systematic review to be included. When systematic reviews reported a broad age range, we included the review if the mean participant age was 65 years or greater and/or if a sub-group analysis was conducted on participants with a mean age of 65 years or greater. Although our focus was specific to LOCs (i.e., home or alternative setting), we included reviews that examined interventions, care options, and service delivery models within the context of eligible LOCs. Ineligible LOCs were acute hospital stays and temporary or transitional placements (e.g., respite care or community/institutional placements for less than 3 months) [15]. If two reviews had $100 \%$ overlap of included studies (e.g., systematic review updates, systematic reviews with the same aims and outcomes), we included the review with the most complete and up-to-date dataset. All eligible LOC comparisons were considered.

\section{Search strategy}

An Information Specialist designed and conducted the search with input from the research team. Our search strategy aimed to find all systematic reviews that compared home care to alternative locations of care. The search included a mix of subject headings and keywords related to the participants (e.g., aged, senior, older, elder, geriatric) and home care (e.g., home care services, home hospitalization). The rationale for searching 'home care' alone was to find all reviews concerning home care and select only those that compare home care to at least one other LOC. Databases, searched from journal inception to June 2016, included: MEDLINE, the Cochrane Library, EMBASE, and Cumulative Index to Nursing and Allied Health Literature (CINAHL). The search included a mix of subject headings and keywords related to the participants (e.g., aged, senior, older, elder, geriatric) and home care (e.g., home care services, home hospitalization). Limits were applied to study designs (e.g. reviews only) and participant characteristics (e.g., not infant, child or adolescent) (Additional file 1). We also scanned the reference list of included systematic reviews for systematic review eligibility. When we were unable to obtain the full text, we emailed the first author to request the paper.

\section{Review selection}

After removing duplicates, two reviewers independently screened citations in three phases: title, abstract, and full text. Title screening was guided by the citations' overall relevance to this study. The eligibility criteria guided the abstract and full text screenings. Reviewers made one of three screening choices: include, exclude, or unsure. Citations were removed from further screening when both reviewers decided the article should be excluded. When at least one reviewer assigned an article to the 'include' or 'unsure' category, the article moved to the next screening stage. Inclusion and exclusion of full text articles required reviewer consensus.

\section{Data collection}

One reviewer extracted data using a standardized pre-piloted form. A second reviewer independently verified all extracted data points for accuracy and completeness. All inconsistencies were resolved through consensus. Extracted variables included: citation information, review objectives, review methodology, review critical appraisal methods, number of studies included, number and characteristics of participants, LOCs, comparator LOCs, interventions, elder health and wellbeing outcomes, synthesis/summary of results, conclusions, and strengths/ limitations. We only extracted information from the systematic reviews that were relevant to this overview [16]. In other words, for some systematic reviews, we only examined a subset of studies (e.g., those related to the elderly or that compared LOCs).

\section{Data synthesis}

We synthesised the data narratively using groupings of LOC comparisons that emerged from the included reviews. Pooling the data was not appropriate due to the heterogeneity in study design, population characteristics, LOCs, interventions, measures, and outcomes, within and across included systematic reviews.

\section{Critical appraisal of included reviews}

Two raters assessed the methodological quality of included systematic reviews using the 11-item Assessing the Methodological Quality of Systematic Reviews (AMSTAR) instrument [17]. Disagreements were resolved through consensus and third person arbitration. A sensitivity analysis was conducted at the systematic review level (i.e., not primary studies). We re-examined higher quality systematic reviews that scored at or above the mean AMSTAR score.

\section{Results}

\section{Review selection}

The search yielded 2,575 citations (Fig. 1). After removing duplicates and screening titles and abstracts, we examined 207 full texts, of which 17 were eligible for inclusion. Screening the reference lists of included systematic reviews revealed 16 potential titles, of which 2 were included. In total, 19 systematic reviews met our inclusion criteria. 


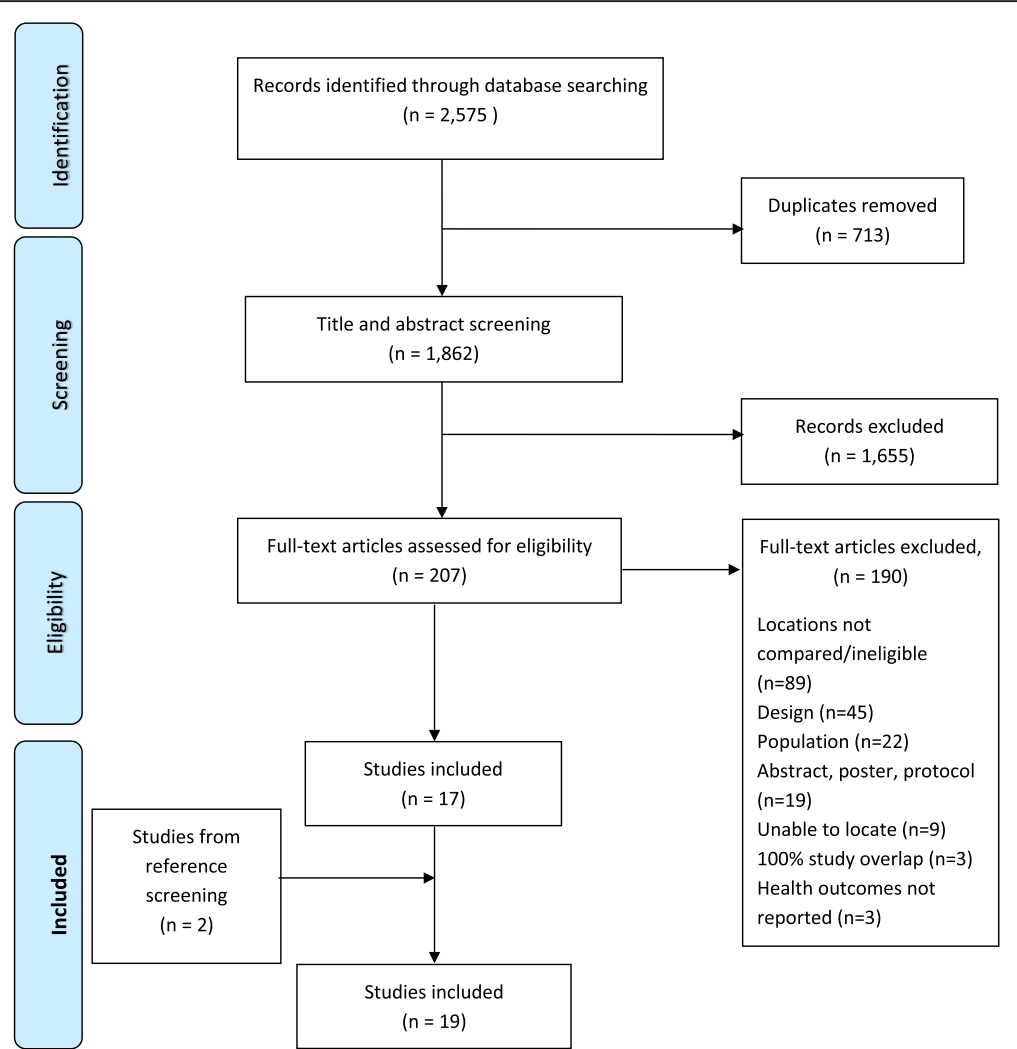

Fig. 1 Flow chart

\section{Characteristics of reviews}

Included systematic reviews were published in English between 2000 and 2016 and originated from seven different countries: United Kingdom $(n=6)$, Canada $(n=5)$, Australia $(n=2)$, the Netherlands $(n=2)$, United States $(n=2)$, Switzerland $(n=1)$, and Sweden $(n=1)$ (Table 2). Three hundred and forty studies (range 1 to 110 ) with a reported 271,660 participants (range 96 to 108,838 ) were synthesized across the systematic reviews. LOC comparisons were: home with support versus independent living at home $(n=11$ reviews), home care versus institutional care ( $n=3$ reviews), and rehabilitation at home versus other conventional rehabilitation services $(n=7)$. Data from the Mehta [18] and Fens [19] systematic reviews were included in two categories.

\section{Summary of findings}

Home with support versus independent living at home ( $n=11$ reviews)

Compared to usual care, typically defined as independent living at home, seven reviews favoured home with support, three found no difference, and one reported insufficient evidence (Tables 3 and 4). Home support by an interdisciplinary team reduced nursing home and hospital admissions, decreased falls, and improved physical function [20]. Two reviews found that preventative home visits for community dwelling elders improved health and functional status, mortality rates, and delayed hospitalization and nursing home use $[21,22]$. However, another review found no difference in health outcomes due to preventative home visits [23]. Transitional support provided by clinical nurse specialists reduced re-hospitalizations [24]. Two reviews examined case management interventions (i.e., collaborative patient care to meet patients' holistic needs). One found delayed onset of nursing home placement and decreased nursing home admissions, length of stay, and community care service use [25]. The other found that case management approaches for people with dementia improved cognitive status and decreased institutionalization and caregiver outcomes at certain time points [26]. One review concluded that home health monitoring technologies reduced undesired health outcomes for the elderly [27]. Another review found limited evidence for the effectiveness of multidisciplinary care for stroke patients at home compared to usual care [19]. Mehta [18] found no difference between receiving home physiotherapy for hip fractures compared to no physiotherapy. Finally, a Cochrane review found little high-grade evidence that physical modifications to the home environment affected the likelihood of sustaining an injury in the home [28]. 


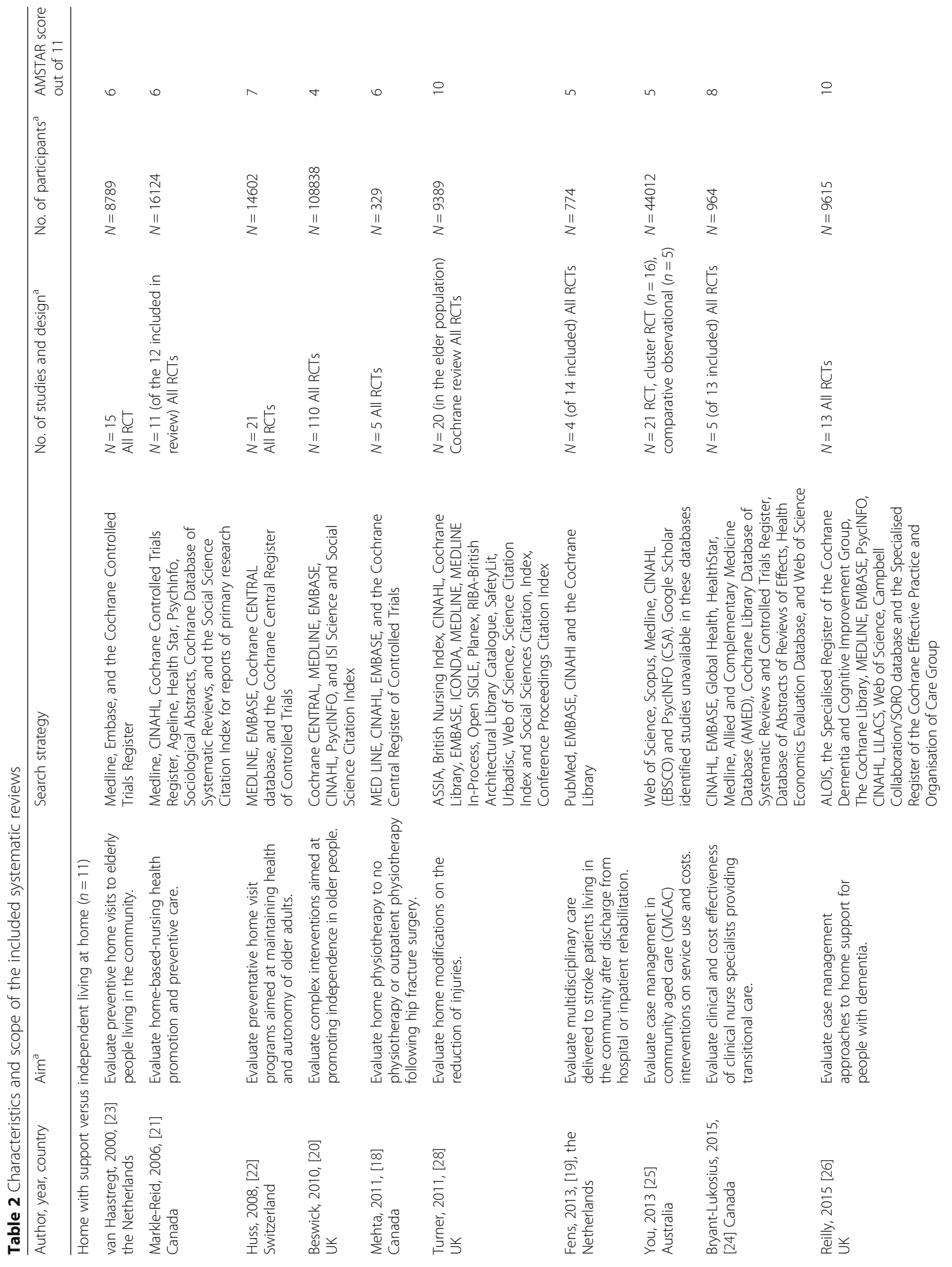




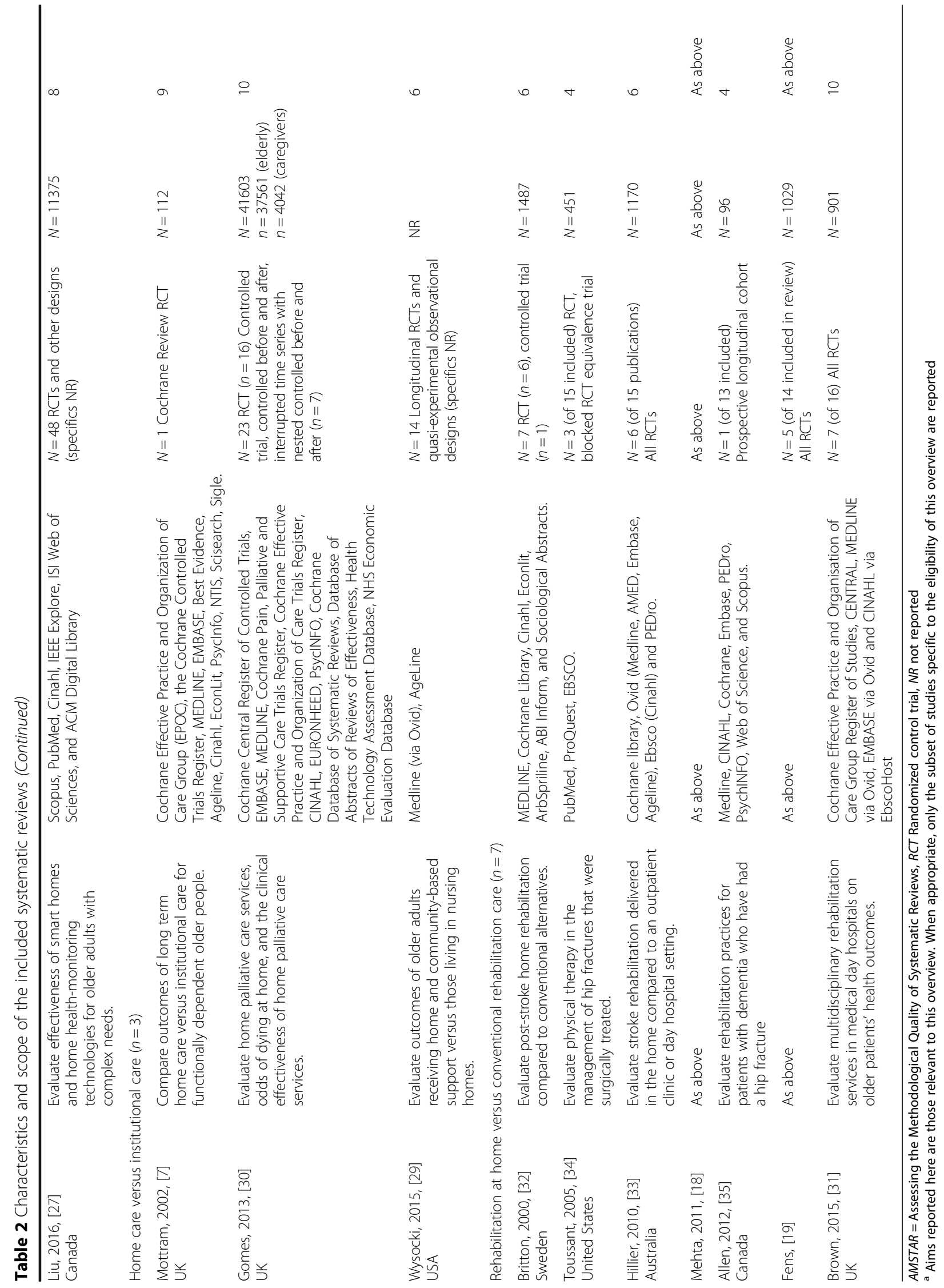




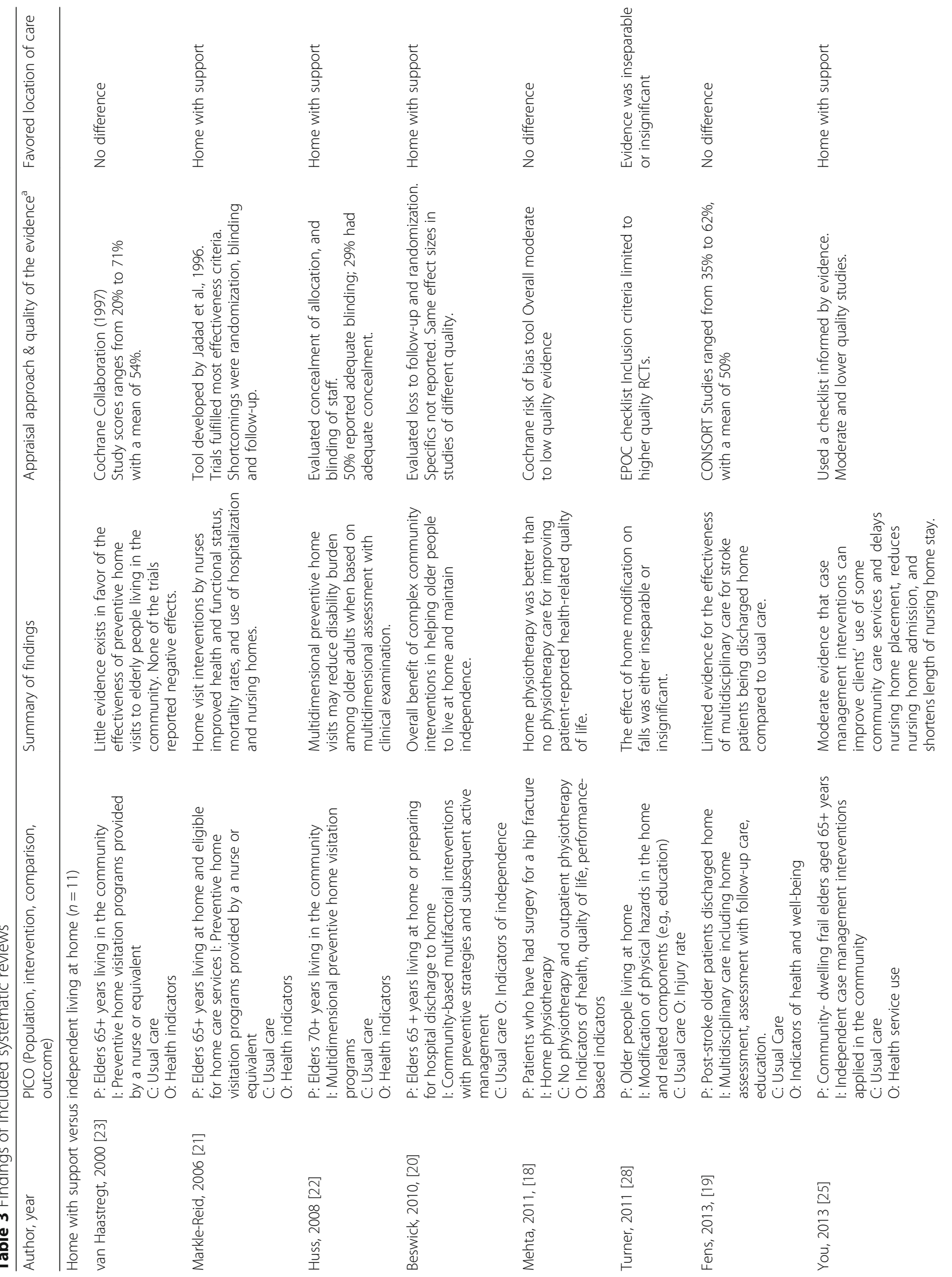




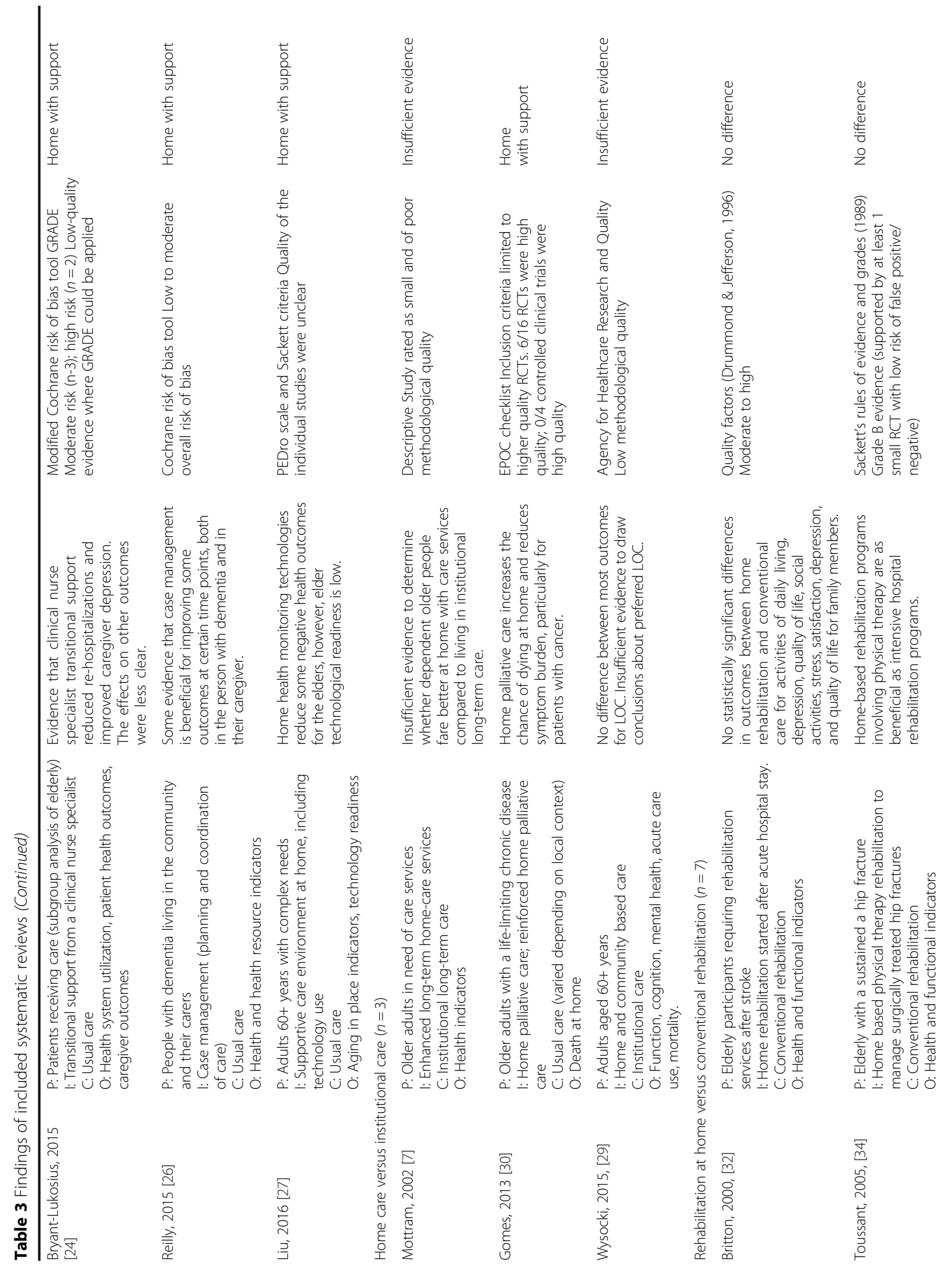




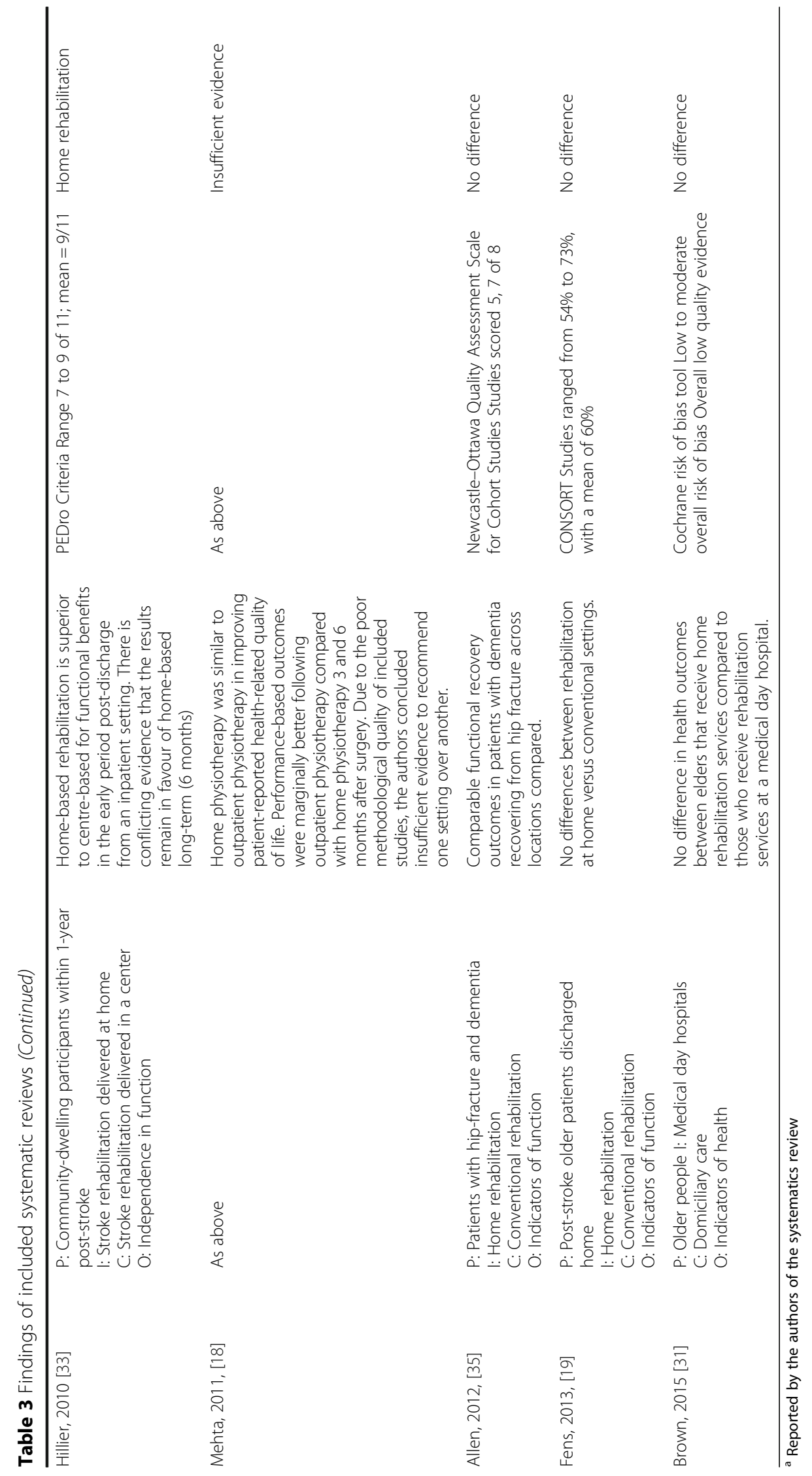




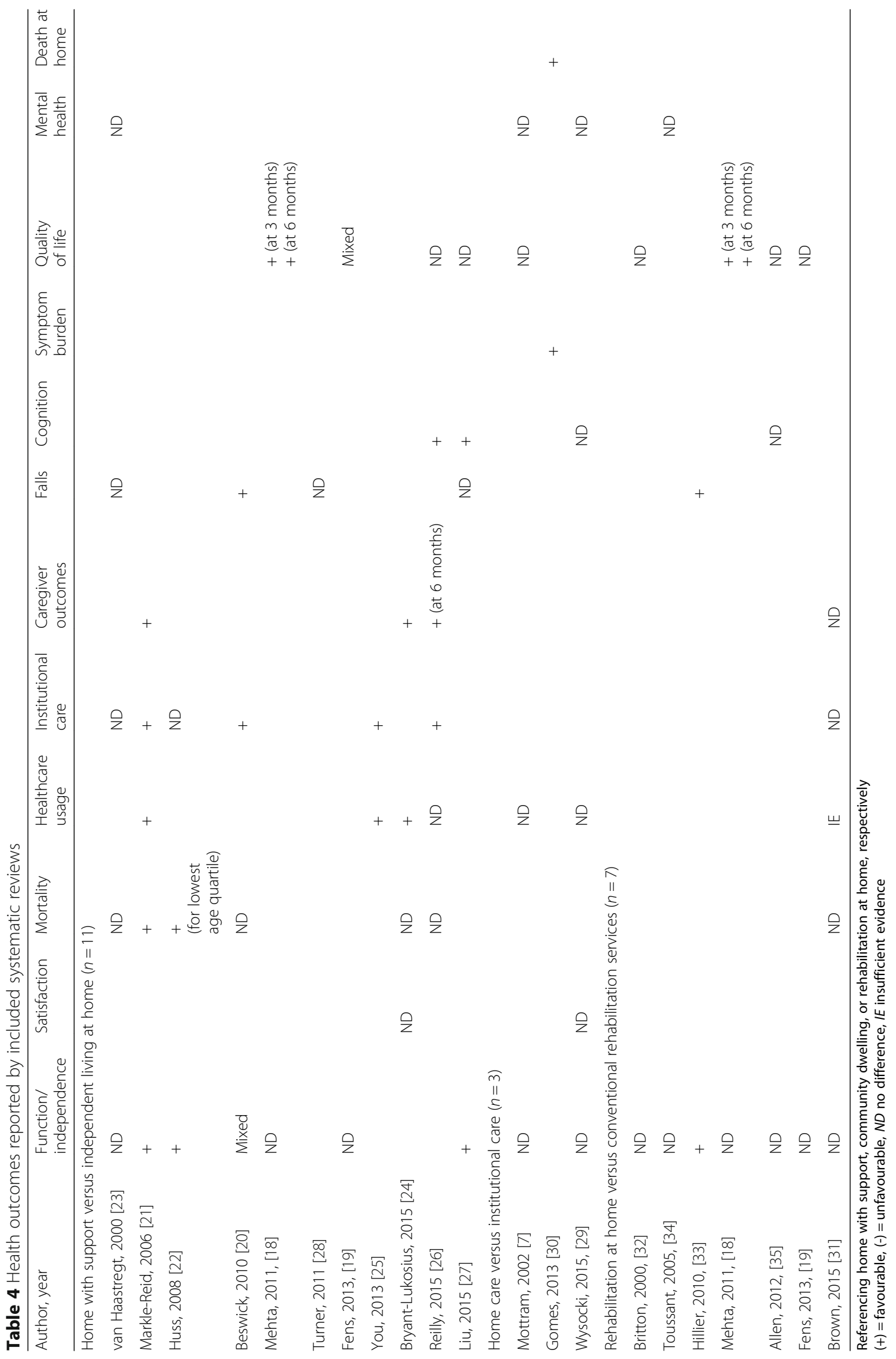




\section{Home care versus institutional care ( $n=3$ reviews)}

Three reviews compared home care to institutional care. Although each review differed in their purpose and scope, two found insufficient evidence, and one review favoured home care (Tables 3 and 4). A Cochrane review that examined the health outcomes of elders receiving home care compared to institutional care found only one randomized controlled trial and concluded that there was insufficient evidence to make recommendations about alternatives to institutional care for the elderly [7]. Similarly, a review that examined various home care options compared to nursing homes for caredependent elders found no significant differences for most health outcomes [29]. Another Cochrane review found that elders who received home palliative care compared to institutional palliative care were more likely to die at home and experience less symptom burden, particularly for patients with cancer [30].

\section{Home rehabilitation versus conventional rehabilitation care ( $n=7$ reviews)}

Within this category, five reviews reported no differences between home and conventional rehabilitation care, one concluded insufficient evidence to make a recommendation, and one reported that home rehabilitation was superior to conventional options in the short-term (Tables 3 and 4). Specifically, one Cochrane review included seven studies that compared homebased rehabilitation to medical day hospital rehabilitation for community dwelling elders [31]. Findings showed no difference between these settings on health outcomes. Three reviews examined post-stroke rehabilitation. In one review, there were no significant differences between home and conventional rehabilitation for activities of daily living, depression, quality of life, and social activities [32]. Pooled evidence from the other review suggested that home-based rehabilitation, provided between 6 and 12 weeks after discharge from inpatient care, might yield superior functional benefit and satisfaction; however, benefits were less clear after 6 months [33]. The third review showed no difference between home and conventional rehabilitation for outcomes related to activities of daily living and quality of life [19].

Three reviews examined rehabilitation after hip fractures. One review found no difference between home and outpatient physiotherapy for patient-reported healthrelated quality of life, but suggested that performancebased outcomes might be marginally better following outpatient services [18]. However, due to the low quality of the primary studies the authors concluded that there was insufficient evidence to recommend one service delivery model over the other. A similar review found that home-based rehabilitation programs involving physical therapy for hip fractures were as beneficial as hospital rehabilitation for patients who had not lost many functional abilities prior to the fracture [34]. The other review examined elders with dementia and hip fractures and found no differences in health outcomes between the settings [35].

\section{Critical appraisal with sensitivity analysis}

Based on the AMSTAR ratings, the average quality of included systematic reviews was moderate, with a mean AMSTAR score of 7 of 11 (median $=6$; range $=4$ to 10) (Table 5). No systematic reviews met the criteria of disclosure of financial interest (Item 11) because none reported funding/support of each included study. We re-examined systematic reviews scoring at or above the mean. In the home with support versus independent living at home category, 5 of 11 reviews were of higher quality. Four reviews, including one Cochrane, which examined preventative home visits, transitional support, case management, and home health technologies, concluded that home with support was superior to independent living at home [22, 24, 26, 27]. The other, a Cochrane review, concluded that inseparable or insignificant results for home modification to reduce falls [28].

Two of three reviews in the home care versus institutional care category were high quality (AMSTAR ratings were 9 and 10 of 11, both Cochrane reviews). The findings, however, were mixed: one review concluded that there was insufficient evidence to determine whether elders fare better with home care compared to institutional care [7] and the other supported home palliative care over institutional palliative care to improve the likelihood of dying at home and decreasing symptom burden [30]. Only the Brown et al., [31] Cochrane review was of high enough quality (AMSTAR score of 10/11) to be included in the home versus conventional rehabilitation category. This review found no significant differences in elder health outcomes between those who received home rehabilitation compared to those who attended medical day hospitals for rehabilitation services.

\section{Discussion}

We synthesized systematic reviews evaluating the impact of home care versus alternative LOCs on elder health outcomes. Overall, we found 19 eligible systematic reviews covering 340 studies and 271,660 participants. Reviews originated from seven industrialised countries that are known to be experiencing rapid growth among their elder population. Categories of comparisons included: home with supports versus independent living at home, home care versus institutional care, and home rehabilitation versus conventional rehabilitation. Most reviews favoured home with support to independent living at home. Results for home care versus institutional care were mixed. Most reviews found no differences in 
Table 5 Authors' judgements about each risk of bias item of included systematic reviews

\begin{tabular}{|c|c|c|c|c|c|c|c|c|c|c|c|c|}
\hline AMSTAR ITEMS & 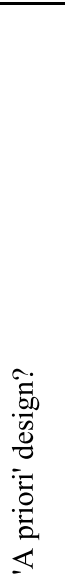 & 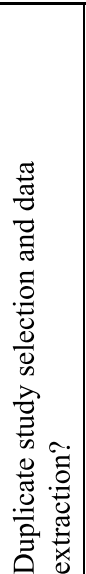 & 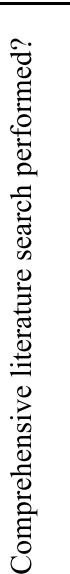 & 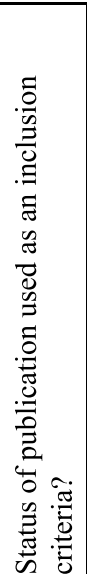 & 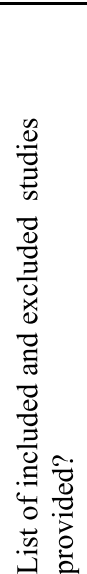 & 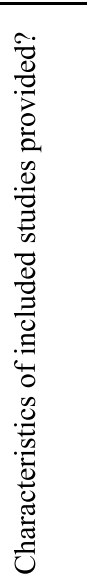 & 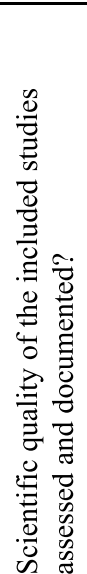 & 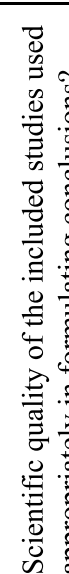 & 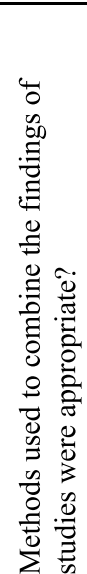 & 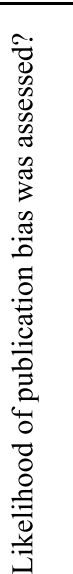 & 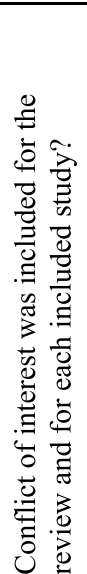 & 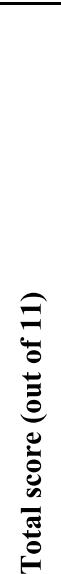 \\
\hline van Haastregt, 2000, [23] & 0 & 0 & 0 & 0 & 0 & 0 & 0 & 0 & 0 & 0 & 0 & 6 \\
\hline Britton, 2000, [32] & 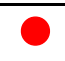 & $\mathrm{O}$ & $\mathrm{O}$ & $\mathrm{O}$ & $\mathrm{O}$ & $\mathrm{O}$ & 0 & ○ & 0 & ○ & 0 & 6 \\
\hline Mottram, 2002, [7] & 0 & 0 & 0 & 0 & 0 & 0 & 0 & 0 & 0 & 0 & 0 & 9 \\
\hline Toussant, 2005, [34] & 0 & 0 & 0 & 0 & 0 & 0 & 0 & 0 & 0 & 0 & 0 & 4 \\
\hline Markle-Reid, 2006, [21] & - & 0 & 0 & 0 & ○ & 0 & 0 & 0 & 0 & - & 0 & 6 \\
\hline Huss, 2008, [22] & 0 & 0 & 0 & 0 & 0 & 0 & 0 & 0 & 0 & 0 & 0 & 7 \\
\hline Beswick, 2010, [20] & 0 & 0 & 0 & 0 & 0 & 0 & $\overline{0}$ & 0 & $\theta$ & 0 & 0 & 4 \\
\hline Hiller, 2010, [33] & 0 & 0 & 0 & 0 & 0 & 0 & 0 & 0 & 0 & 0 & 0 & 6 \\
\hline Mehta, 2011, [18] & 0 & 0 & 0 & 0 & 0 & 0 & 0 & 0 & 0 & 0 & 0 & 6 \\
\hline Turner, 2011, [28] & 0 & 0 & 0 & 0 & 0 & 0 & 0 & 0 & 0 & 0 & 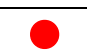 & 10 \\
\hline Allen, 2012, [35] & 0 & (9) & 0 & 0 & 0 & 0 & 0 & 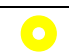 & 0 & $\overline{0}$ & $\overline{0}$ & 4 \\
\hline Fens, 2013, [19] & 0 & 0 & 0 & 0 & 0 & 0 & 0 & 0 & 0 & 0 & 0 & 5 \\
\hline Gomes, 2013, [30] & 0 & 0 & 0 & 0 & 0 & 0 & 0 & 0 & 0 & 0 & 0 & 10 \\
\hline You, 2013, [25] & 0 & 0 & 0 & 0 & 0 & 0 & 0 & 0 & 0 & 0 & 0 & 5 \\
\hline $\begin{array}{l}\text { Bryant-Lukosius, 2015, } \\
{[24]}\end{array}$ & 0 & 0 & 0 & 0 & 0 & 0 & 0 & 0 & 0 & 0 & 0 & 8 \\
\hline Liu, 2015, [27] & 0 & 0 & 0 & 0 & 0 & 0 & 0 & 0 & 0 & 0 & 0 & 7 \\
\hline Reilly, 2015, [26] & $\mathrm{O}$ & $\mathrm{O}$ & $\mathrm{O}$ & $\mathrm{O}$ & 0 & $\mathrm{O}$ & 0 & $\mathrm{O}$ & 0 & $\mathrm{O}$ & 0 & 10 \\
\hline Wysocki, 2015, [29] & 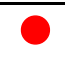 & $\mathrm{O}$ & 0 & $\mathrm{O}$ & 0 & 0 & 0 & $\mathrm{O}$ & 0 & 0 & 0 & 6 \\
\hline Brown, 2015, [31] & 0 & 0 & 0 & 0 & 0 & 0 & 0 & 0 & 0 & 0 & 0 & 10 \\
\hline
\end{tabular}

\section{$\mathrm{O}=$ yes}

$=$ no

= unsure

health outcomes between rehabilitation at home versus conventional rehabilitation services. The quality of included systematic reviews was moderate. Our results lead us to make the following observations.

First, as aging in a desirable LOC may contribute to overall health and wellbeing in the late-life period
[36, 37], we were not surprised to find several reviews that examined LOC as a function of elder health and wellbeing. Many elderly people prefer and choose to age at home [38-42]. Healthcare organizations and policy makers are increasingly challenged to better support a shift from institutionalized long-term care 
to support elders to remain in their community [43]. The included reviews indicate a growing literature of interventions designed to promote health, function and independent living among community dwelling elders. Yet, many older adults anticipate moving, often to a more institutionalized location. Even those who do not anticipate moving might be compelled to move due to unforeseen life changes (e.g. loss of a spouse, illness) [40, 44]. As life expectancy increases and more people in late life suffer from multimorbidity, their relocation will represent a significant market for new residential options beyond the limited choices of private nursing homes or public long-term care facilities [45]. Therefore, an improved understanding of the role of long-term LOCs on elders' health and wellbeing is central to any strategy aimed at fostering elders' quality of life [46].

Second, the impact of home care compared to institutional care on elder health outcomes was less clear. The Mottram et al. [7] and Wysocki et al. [29] reviews had similar PICOS questions, and despite the 13-year publication gap and new research, both concluded that there was insufficient evidence to draw firm conclusions. Similarly, most systematic reviews that examined location of rehabilitation services and elder health outcomes found no differences or insufficient evidence. Inconclusive findings suggest that informed patient preferences and individual needs should guide elders' decision to move from home to an alternative LOC. Examination of LOC options and preferences should also incorporate contextual factors, such as the quality of available services or other interventions. Consistent the ecological model of aging and person-environment fit, elders' specific needs (e.g., mobility, cognitive, social) must be evaluated and matched to available LOC options with appropriate interventions or services to address those needs.

Third, our results highlight that most home care research focuses on few LOC comparisons. Further, most systematic reviews did not compare across multiple potential LOCs. When examined individually, each comparison provided insight regarding potential outcomes of one LOC option compared to the other. However, it was not possible to integrate the findings to determine the best LOC based on elder health outcomes. Although we did not examine the primary studies, our overview identifies a need for better research targeting long-term LOC options for the late-life years. Improved research and additional investment will translate into more rigorous methods and generate a more complete understanding of the impact of LOCs on elders' health.

Our findings should be interpreted within the context of its limitations. First, our search strategy focused on home care, thus terms such as 'day hospital' and 'assisted living' were not included. Also, we did not search the grey literature. Therefore, we might have missed eligible systematic reviews. Second, several factors made this literature challenging to synthesize. We found considerable heterogeneity within and across included systematic reviews regarding population characteristics, locations considered, measures used, and outcomes reported, precluding firm conclusions. LOCs were also poorly and inconsistently described in the literature. Development of an accepted taxonomy for LOCs would help advancement in this field. Third, the study designs of the included reviews made direct comparisons for LOCs difficult and we noted a risk of indication bias (elders' health status correlates with the intervention and is a risk indicator for the outcome) within several of the primary studies. Causal relationships between LOCs and elder health outcomes could not be measured. Instead, this overview provides a broad summary of the state of knowledge regarding elder home care compared to alternative LOCs and elder health outcomes. Fourth, there was some primary study overlap across the systematic reviews, potentially over-representing certain findings. However, the purpose of an overview of systematic reviews is to provide a high level description of the state of the evidence [16]. To offset this limitation, we excluded studies with $100 \%$ of primary study overlap. Finally, new and relevant studies might have been excluded if they were not yet included in a systematic review.

\section{Conclusions}

Results from this overview of systematic reviews, which evaluated the impact of home care compared to alternative long-term LOCs on elder health outcomes, suggests that home interventions and/or supports that promote elder health and independence might be effective in helping elders age at home. However, we are unable to make recommendations regarding the impact of other LOCs compared to home on elders' health and wellbeing. Without a more robust evidence base, elders considering moving from home will be unable to make evidence informed decisions about which long-term alternative LOC to choose that fits best with their needs and preferences.

\section{Additional file}

\section{Additional file 1: Search strategy for Medline. (DOCX $13 \mathrm{~kb}$ )}

\section{Abbreviation}

LOC: Location of care

\section{Acknowledgements}

William Witteman is the information technologist who designed and conducted the search strategy. We are also thankful to Roxanne Lepine, information technologist, for her ongoing support. 


\section{Funding}

This research was funded by Canadian Frailty Network (known previously as Technology Evaluation in the Elderly Network, TVN), supported by Government of Canada through Networks of Centres of Excellence (NCE) Program, and by the Ministère de la Santé et des Services Sociaux (MSSS) du Québec. At the time of this study, we also had the support of the following local health networks: the Consortium InterESt Santé, the CISSS de la Gaspésie and the CIUSSS de la Capitale Nationale and the Agence de la Santé et des Services sociaux de la Capitale-Nationale. Three local health networks also supported us: the Consortium InterESt Santé, the CSSS du Rocher-Percé and the CIUSSS de la Capitale-Nationale. Since April $1^{\text {st }}$ 2015, these organizations have merged within larger organizations. We also had funding from the Quebec Ministère de la Santé et des Services sociaux (MSSS). LB is supported by a Queen Elizabeth II Graduate Scholarship in Science and Technology. MG is supported by a postdoctoral scholarship awarded by the Canadian Institutes of Health Research (CIHR), Funding Reference Number MFE-140842. DM is supported by CFN and the University of Ottawa, department of Anesthesiology. FL is Tier 1 Canada Research Chair in Shared Decision Making and Knowledge Translation at Université Laval. DS is supported by a University Research Chair in Knowledge Translation to Patients. The sponsors had no role in the design or conduct of the study, nor in collection, management, analysis, or interpretation of the data; nor in the preparation, review, approval of the manuscript or decision to submit it for publication

\section{Availability of data and materials}

The supporting data are publically available and can be viewed by obtaining the systematic reviews included in this overview.

\section{Authors' contributions}

All authors participated in the design of the study. LB was the primary investigator responsible for all parts of the study and drafted the manuscript. FL and DS were the senior authors. LB, DM, MP, DS and MM participated in citation screening. LB, DM GP and MG were involved in data extraction and appraisal. All authors read, edited and approved the final manuscript.

\section{Competing interests}

The authors declare that they have no competing interests.

\section{Consent for publication}

Not applicable.

\section{Ethics approval and consent to participate}

Not applicable.

\section{Author details \\ 'Population Health, Faculty of Health Sciences, University of Ottawa, 25 University Private, Ottawa, ON K1N 7 K4, Canada. ${ }^{2} \mathrm{CHU}$ de Québec Research Centre-Université Laval site Hôpital St-Francois d'Assise, 10 Rue Espinay, Quebec G1L 3 L5, Canada. ${ }^{3}$ Department of Anesthesiology, Faculty of Medicine University of Ottawa, The Ottawa Hospital, 1053 Carling Ave, Rm B311, Ottawa, ON K1Y 4E9, Canada. ${ }^{4}$ Centre de santé et de services sociaux de la Vieille-Capitale, 880, rue Père-Marquette, Québec G1M 2R9, Canada. ${ }^{5}$ Centre intégré universitaire en santé et services sociaux de la Capitale-Nationale, 880, rue Père-Marquette, Québec G1M 2R9, Canada. ${ }^{6}$ Ottawa Hospital Research Institute, 501 Smyth Road, Ottawa, ON K1H 8L6, Canada. ${ }^{7}$ University of Ottawa, 451 Smyth Road, Ottawa, ON K1H 8 M5, Canada.}

Received: 18 May 2016 Accepted: 9 December 2016

Published online: 14 January 2017

\section{References}

1. Bynum JPW, Meara ER, Chang CH, Rhoads JM, Bronner KK. Our parents, ourselves: health care for an aging population: a report of the Dartmough Atlas Project. Lebanon, NH: The Dartmouth Institute of Health Policy \& Clinical Practice; 2016.

2. United Nations. World Population Ageing 2013. Department of Economic and Social Affairs Population Division 2013

3. Ausserhofer D, Deschodt M, De Geest $S$, van Achterberg T, Meyer G, Verbeek H, Sjetne IS, Malinowaska-Lipien I, Griffiths P, Schliter W, et al. "There's No Place Like Home": A Scoping Review on the Impact of Homelike
Residential Care Models on Resident-, Family-, and Staff-Related Outcomes. J Am Med Dir Assoc. 2016;17(8):685-93.

4. Lawton MP, Nahemow L. Ecology and the aging process. Washington, DC, USA: American Psychological Association; 1973. p. 619-74.

5. Beard J, Officer A, Cassels A. World report on ageing and health. Geneva: World Health Organization; 2015. Available at: http://www.who.int/ageing/ events/world-report-2015-launch/en/. Accessed 30 Nov 2016.

6. Institute of Medicine (US) Committee on Quality of Health Care in America. Crossing the quality chasm: a new health system for the 21 st century. Washington DC; National Academies Press: 2001. Retrieved from: https://www. nationalacademies.org/hmd/ /media/Files/Report\%20Files/2001/Crossing-theQuality-Chasm/Quality\%20Chasm\%202001\%20\%20report\%20brief.pdf. Accessed 30 Nov 2016

7. Mottram P, Pitkala K, Lees C. Institutional versus at-home long term care for functionally dependent older people. Cochrane Database Syst Rev. 2002;1, CD003542.

8. Caron CD, Ducharme F, Griffith J. Deciding on institutionalization for a relative with dementia: the most difficult decision for caregivers. Can J Aging. 2006;25(2):193-205

9. Légaré F, Brière N, Stacey D, Bourassa $H$, Desroches S, Dumont S, Fraser K, Freitas A, Rivest LP, Roy L. Improving Decision making On Location of Care with the frail Elderly and their caregivers (the DOLCE study): study protocol for a cluster randomized controlled trial. Trials. 2015;16(1):567.

10. Grant M, Booth A. A typology of reviews: an analysis of 14 review types and associated methodologies. Health Info Libr J. 2009;26(2):91-108.

11. Moher D, Liberati A, Tetzlaff J, Altman DG, PRISMA group. Preferred reporting items for systematic reviews and meta-analyses: the PRISMA statement. Int J Surg. 2010;8(5):336-41. doi:10.1016/j.jjsu.2010.02.007.

12. National Institute for Health Research. PROSPERO International prospective register of systematic reviews: Inclusion criteria. Retrieved from: http://www.crd. york.ac.uk/prospero/about.php?about=inclusioncriteria. Accessed 30 Nov 2016.

13. Becerra Perez MM, Émond J, Boland L, Brière N, Garvelink M, Freitas A, Thiébaut C, Stacey D, Légaré F. Location of care impact on frail elderly and caregiver health outcomes: A scoping of systematic reviews. Toronto, Ontario: 2nd Annual TVN Conference on Improving Care for the Frail Elderly; 2014.

14. Center for Reviews and Dissemination, University of York. Undertaking Systematic Reviews of Research on Effectiveness. Research report. CRD Report 2001, 4(2). York, UK: NHS Center or Reviews and Dissemination; 2001.

15. Centers for Medicare \& Medicaid Services. Quality Measure Identification Number by CMS Reporting Module. MDS 3.0 User's Manual V8.0 2013, retrieved from: https:/www.cms.gov/medicare/quality-initiatives-patient-assessment-instruments/ nursinghomequalityinits/downloads/quality-measure-identification-number-bycms-reporting-module-table-v12.pdf. Accessed 30 Nov 2016.

16. Smith V, Devane D, Begley CM, Clarke M. Methodology in conducting a systematic review of systematic reviews of healthcare interventions. Medical Research Methodology. 2011;11(1):15. doi:10.1186/1471-2288-11-15.

17. Shea BJ, Grimshaw JM, Wells GA, Boers M, Andersson N, Hamel C, Porter AC, Tugwell P, Bouter. Development of AMSTAR: A measurement tool to assess the methodological quality of systematic reviews. BMC Med Res Methodol. 2007;7:10. doi:10.1186/1471-2288-7-10.

18. Mehta SP, Roy JS. Systematic review of home physiotherapy after hip fracture surgery. J Rehabil Med. 2011;43:477-80.

19. Fens M, Vluggen $T$, van Haastregt JC, Verbunt $V$, Beusman $G H$, van Heugten C. Multidisciplinary care for stroke patients living in the community: a systematic review. J Rehabil Med. 2013;45:321-30.

20. Beswick AD, Gooberman HR, Smith A, Wylde V, Ebrahim S. Maintaining independence in older people. Reviews in Clinical Gerontology. 2010;20:128-53.

21. Markle-Reid M, Browne G, Weir G, Gafni A, Roberts J, Henderson SR. The effectiveness and efficiency of home-based nursing health promotion for older people: a review of the literature. Med Care Res Rev. 2006;63(5):531-69.

22. Huss A, Stuck AE, Rubenstein LZ, Egger M, Clough-Gorr KM. Multidimensional preventive home visit programs for community-dwelling older adults: a systematic review and meta-analysis of randomized controlled trials. J Gerontol. 2008;63A(3):298-307.

23. van Haastregt J, Diederiks J, van Rossum E, Witte L, Witte L, Crebolder HF. Effects of preventive home visits to elderly people living in the community: systematic review. BMJ. 2000;320:754-8.

24. Bryant-Lukosius D, Carter N, Reid K, Donald F, Martin-Misener R, Kilpatrick K, Harbman P, Kaasalainen S, Marshall D, Charbonneau-Smith R, et al. The clinical effectiveness and cost-effectiveness of clinical nurse specialist-led hospital to home transitional care: a systematic review. J Eval Clin Pract. 2015;21(5):763-81. 
25. You E, Dunt D, Doyle C. Case managed community aged care: what is the evidence for effects on service use and costs? J Aging Health. 2013;25(7):1204-42.

26. Reilly S, Miranda-Castillo C, Malouf R, Hoe J, Toot S, Challis D, Orrell M. Case management approaches to home support for people with dementia (Review). The Cochrane Library. 2015;1, CD008345.

27. Liu Y, Stroulia E, Nikolaodis I, Miguel-Cruz A, Rincon AR. Smart homes and home health monitoring technologies for older adults: A systematic review. Int J Med Inform. 2016;91:44-59. 26.

28. Turner $\mathrm{S}$, Aurthur $\mathrm{G}$, Lyons RA, et al. Modification of the home environment for the reduction of injuries (Review). The Cochrane Library. 2011;2, CD003600.

29. Wysocki A, Butler M, Kane RL, Kane RA, Shippee T, Sainfort F. Long-term services and supports for older adults: a review of home and communitybased services versus institutional care. Joural of Aging Social Policy. 2015;27(3):255-79.

30. Gomes B, Calanzani N, Curiale V, McCrone P, Higginson IJ. Effectiveness and cost-effectiveness of home palliative care services for adults with advanced illness and their caregivers (Review). The Cochrane Library. 2013;6, CD007760.

31. Brown L, Forster A, Young J, Crocker T, Benham A, Langhorne P, Day Hospital Group. Medical day hospital care for older people versus alternative forms of care (Review). The Cochrane Library. 2015;6.

32. Britton $\mathrm{M}$, Anderson A. Home rehabilitation after stroke: reviewing the scientific evidence on effects and costs. Int J Technol Assess Health Care. 2000;16(3):842-8

33. Hillier $\mathrm{S}$, Inglis-Jassiem G. Rehabilitation for community-dwelling people with stroke: home or centre-based? A systematic review. Int I Stroke. 2010; 5(3):178-86.

34. Toussant EM, Kohia M. A critical review of literature regarding the effectiveness of physical therapy management of hip fracture in elderly persons. J Gerontol. 2005;60(10):1285-91.

35. Allen J, Koziak A, Buddingh S, Liang J, Buckingham J, Beaupre LA. Rehabilitation in patients with dementia following hip fracture: a systematic review. Bone Health. 2012;64(2):190-201.

36. Hays JC. Living arrangements and health status in later life: A review of recent literature. Public Health Nurs. 2002;19(2):136-51.

37. Somenahalli S, Shipton M. Examining the distribution of the elderly and accessibility to essential services. 2nd Conference of Transportation Research Group of India (2nd Ctrg). 2013;104:942-51.

38. AARP. Fixing to stay: A national survey on housing and home modification issues. American Association of Retired Persons, Washington DC. 2000. Retrieved from: https://assets.aarp.org/rgcenter/il/home_mod.pdf. Accessed 30 Nov 2016.

39. Senior ML, Webster CJ, Blank NE. Residential relocation and sustainable urban form: Statistical analyses of owner-occupiers' preferences. International Planning Studies. 2006:11(1):41-57.

40. Lord S, Després C, Ramadier T. When mobility makes sense: A qualitative and longitudinal study of the daily mobility of the elderly. Journal of Environmental Psychology. 2011;31(1):52-61.

41. Judd B, Liu E, Easthorpe H, Davy L, Bridge C. Downsizing amongst older Australians. Melbourne: AHURI Final Report No. 214, Australian Housing and Urban Research Institute Limited; 2014.

42. Menec V, Bell S, Nocek S, Minnigaleeva GA, Morales E, Ouma T, Parodi JF, Winterton R. Making rural and remote communities more age-friendly: experts' perspectives on issues, challenges, and priorities. J Aging Soc Policy. 2015;27(2):173-91.

43. Williams PA, Lum J, Morton-Chang F, Kuluski K, Peckham A, Warrick N, Ying A. Integrating long-term care into a community-based continuum: shifting from "beds" to "places", IRPP study 59. Montreal, Canada: Institute for Research on Public Policy; 2016.

44. Lord S, Després C. Vieillir en banlieue nord-américaine: le rapport à la ville des personnes âgées. Gérontologie et société. 2011;136(1):189-204.

45. Johnson RW, Toohey D, Wiener JM. Meeting the long-term care needs of the baby boomers. How changing families will affect paid helpers and institutions. Washington, DC: Urban Institute. Project TR; 2007.

46. Edmonston B, Lee S. Residential mobility of elderly Canadians: trends and determinants. Can J Aging. 2014;33(4):378-99.

\section{Submit your next manuscript to BioMed Central and we will help you at every step:}

- We accept pre-submission inquiries

- Our selector tool helps you to find the most relevant journal

- We provide round the clock customer support

- Convenient online submission

- Thorough peer review

- Inclusion in PubMed and all major indexing services

- Maximum visibility for your research

Submit your manuscript at www.biomedcentral.com/submit 